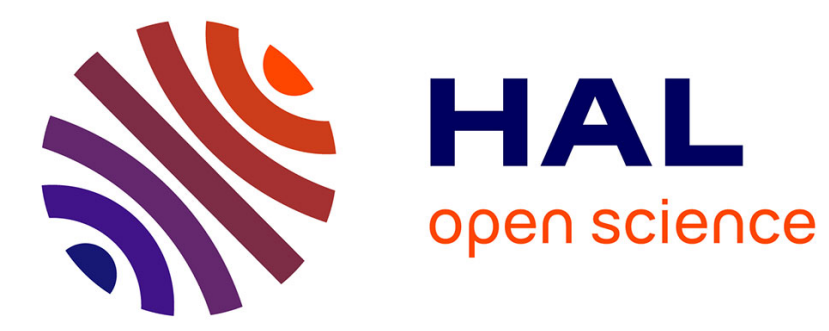

\title{
Transformations microstructurales lors de la mécanosynthèse de l'alliage Ti-47.5A1-3Cr
}

V. Jacob, J. Mishurda, A. Frefer, R. Baccino, F. Moret, C. Suryanarayana, F.

Froes

\section{- To cite this version:}

V. Jacob, J. Mishurda, A. Frefer, R. Baccino, F. Moret, et al.. Transformations microstructurales lors de la mécanosynthèse de l'alliage Ti-47.5A1-3Cr. Journal de Physique IV Proceedings, 1994, 04 (C3), pp.C3-243-C3-250. 10.1051/jp4:1994333 . jpa-00252530

\section{HAL Id: jpa-00252530 https://hal.science/jpa-00252530}

Submitted on 1 Jan 1994

HAL is a multi-disciplinary open access archive for the deposit and dissemination of scientific research documents, whether they are published or not. The documents may come from teaching and research institutions in France or abroad, or from public or private research centers.
L'archive ouverte pluridisciplinaire HAL, est destinée au dépôt et à la diffusion de documents scientifiques de niveau recherche, publiés ou non, émanant des établissements d'enseignement et de recherche français ou étrangers, des laboratoires publics ou privés. 


\title{
Transformations microstructurales lors de la mécanosynthèse de l'alliage Ti-47.5Al-3Cr
}

\author{
V. JACOB, J. MISHURDA* , A. FREFER ${ }^{*}$, R. BACCINO, F. MORET, C. SURYANARAYANA* et \\ F.H. FROES* \\ CEREM/Laboratoire de Génie des Matériaux, CENG 85X, 38041 Grenoble cedex, France \\ * IMAP/College of Mines \& Earth Resources, University of Idaho, Moscow, Idaho 83843-4195, U.S.A.
}

\begin{abstract}
Mechanical milling of pre-alloyed $\mathrm{Ti}-47.5 \mathrm{Al}-3 \mathrm{Cr}$ (at.\%) powder has been carried out in a vibratory mill. The microstructural evolution of the materials was studied as a function of milling time. An ultrafine microstructure was produced with a crystallite size of about $15 \mathrm{~nm}$ and a partial amorphization was obtained after 2 hours of milling.
\end{abstract}

\section{Introduction}

Les alliages à base d'aluminiures de titane TiAl $(\gamma)$ et $\mathrm{Ti}_{3} \mathrm{Al}\left(\alpha_{2}\right)$ ont été très étudiés car ils présentent un grand intérêt pour des applications structurales à haute température en aéronautique (faible densité, résistance spécifique élevêe, résistance à haute température, résistance au fluagel mais leur développement a été jusqu'ici limité par leur fragilité à basse température. Les propriétés mécaniques de ces matériaux peuvent être améliorées par addition d'un troisième élément $(\mathrm{Cr}, \mathrm{Nb}, \mathrm{V}, \mathrm{Mn}, \mathrm{Mo}, \mathrm{Ta} . .$.$) ou par modifications$ microstructurales laffinement de la taille de grain, homogénéisation, transformations de phases, précipitation...) [1-3]. De telles transformations peuvent être obtenues par mécanosynthese, un procédé de la métallurgie des poudres [4].

Lors de la mécanosynthèse, les poudres sont à plusieurs reprises soudées, fracturées et ressoudées dans un broyeur énergétique $[5,6]$. Cela conduit à un mélange intime à une échelle extrêmement fine avec la possibilité de former une variété de phases a l'équilibre et hors équilibre telles que des solutions solides sursaturées, des phases intermédiaires cristallisées et métastables et des phases amorphes. Les matériaux élaborés par mécanosynthèse sont aussi caractérisés par une matrice à taille de grains submicronique. Des renforts de taille nanométrique (oxydes, carbures...) peuvent être introduits dans le matériau soit par addition directe de poudre céramique soit par réaction avec un adjuvant. Le rôle des dispersoïdes est d'éviter le grossissement de grain à haute température et d'améliorer les propriétés de fluage [7].

Un travail considérable a été mené concernant la mécanosynthèse du système Ti-Al. La formation d!une phase amorphe a été étudiée en fonction de la composition de l'alliage et du temps de broyage [8]. Suryanarayana et al. ont montré la formation du composé intermétallique TiAl par broyage de deux composés fragiles $\mathrm{Al}_{3} \mathrm{Ti}$ and $\mathrm{TiH}_{2}$ [9]. Ils ont suggéré que la mécanosynthèse peut améliorer la ductilité de l'alliage Ti-24Al-11 Nb (at.\%) en permettant l'affinement de la taille de grain et la synthèse de la phase B2 (cubique centré) [10].

L'objectif du présent travail est d'étudier les transformations à l'état solide intervenant lors de la mécanosynthèse d'un alliage Ti-47.5Al-3Cr (at. \%) élaboré par solidification rapide. 


\section{Expériences}

Broyage. La poudre initiale a été obtenue par atomisation gazeuse de l'alliage $\mathrm{Ti}-47.5 \mathrm{Al}-3 \mathrm{Cr}$ (at.\%), elle est sphérique avec une granulométrie de $150 \mu \mathrm{m}$.

Le broyage a été réalisé dans un broyeur vibrant du type SPEX mill avec des billes en acier de diamètre $4.76 \mathrm{~mm}$ en une atmosphere d'argon. Le rapport massique bille sur poudre est de 10:1. Un adjuvant de broyage ( 1 pds. \% d'acide stéarique) a été ajouté pour limiter l'agglomération des poudres. Des durées de broyage de 6 minutes à 4 heures ont été étudiés. La quantité de poudre initiale est de 5 grammes.

Caractérisation. L'évolution microstructurale des poudres lors de la mécanosynthèse a été étudiée par diffraction RX (radiation Co $K_{\alpha}$ ), MEB, microsonde électronique, MET et microanalyse par spectroscopie par dispersion d'énergie des photons $X$.

Les examens en microscopie en transmission ont été réalisés directement sur les grains de poudre ou sur des échantillons préparés par ultramicrotomie. Cette technique consiste à enrober des grains de poudre dans une résine qui est ensuite découpée en lames de $80 \mathrm{~nm}$ d'épaisseur avec un couteau diamant [11]. L'ultramicrotomie a été utilisée uniquement pour préparer les échantillons de microanalyse car elle endommage les matériaux étudiés.

\section{Résultats}

Observations métallurgiques. Les morphologies des poudres initiale et broyées ont été observées par microscopie électronique à balayage (fig.1). Les poudres broyées sont constituées de particules aplaties dont la taille décroît avec le temps de broyage jusqu'à quelques micromètres.

La poudre initiale présente une structure biphasée. Les images en électrons rétrodiffusés des poudres broyées $n^{\prime}$ indiquent pas de contraste de composition dû à des ségrégations contrairement à la poudre initiale.

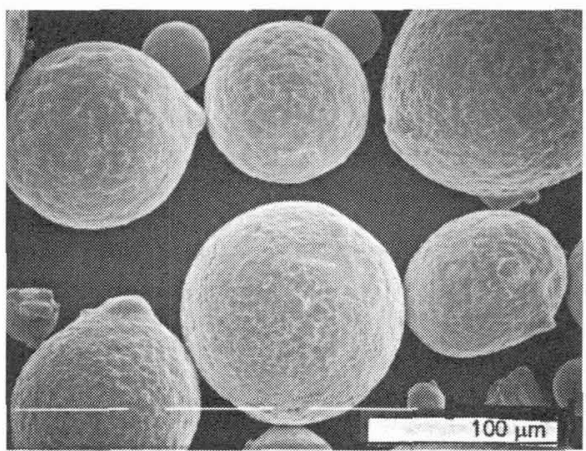

Fig. 1 - a) MEB : poudre initiale

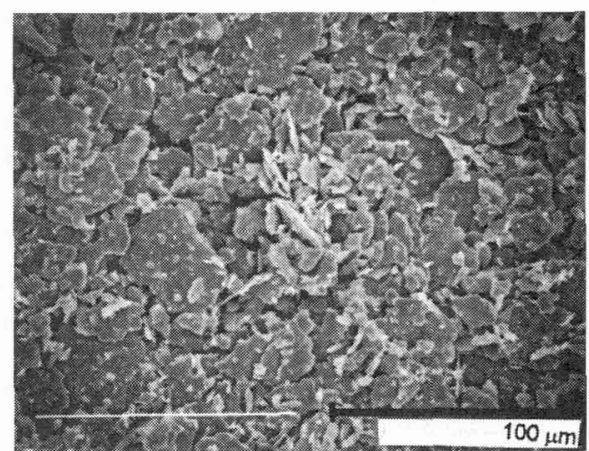

b) MEB : Poudre broyée 1 heure

Les analyses par microsonde électronique montrent une distribution homogène du titane dans chaque phase. Cependant, quelques régions présentent un léger appauvrissement en titane associé à une augmentation des teneurs en aluminium et en chrome. Ces régions correspondent certainement à la phase TiAl. L'homogénéité en composition des poudres broyées a été mise en évidence par microanalyse sur les coupes réalisées par ultramicrotomie.

Les images MET en champ sombre ont montré que la taille des cristallites (ou domaines cohérents) n'est pas homogène dans la poudre initiale et varie de 20 à $150 \mathrm{~nm}$. La taille des cristallites décroît jusqu'à 10-15 nm après 6 minutes de broyage (annexe fig.1\}. Pour des durées supérieures, aucun affinement supplémentaire n'a été observé (annexe fig.2). La structure microcristallisée de ces poudres a été mise en évidence par des clichés de diffraction (annexe fig.3). Les observations au MET des poudres broyées 2 et 4 heures a été 
difficile du fait de l'instabilité de la structure : grossissement de grains et mise en ordre ont lieu sous le faisceau d'électrons. L'existence d'une phase amorphe est mise en évidence par diffraction sur la poudre broyée 2 heures et 4 heures (annexe fig.3-c). De petits précipités répartis de manière homogène ont été observés sur les poudres broyées.

Diffraction RX. La figure 2 montre les spectres de diffraction RX des poudres initiale et broyées 11 et 4 heures). La poudre initiale a une structure biphasée $\gamma+\alpha_{2}$ dans laquelle TizAl est la phase majoritaire. Aucune raie de surstructure n'est observée, ce qui indique un état désordonné et la présence possible de la phase $\alpha$. Au cours du broyage, un élargissement continu des pics TiAl et Ti 3 Al est observé. Après 2 heures de broyage, un épaulement correspondant a la phase amorphe apparait sous les pics (200), (002), (201) de $\mathrm{Ti}_{3} \mathrm{Al}$ et le pic (111) de TiAl (annexe fig.4).

Les spectres de diffraction de RX ont été déconvolués pour séparer les contributions des intensités ITIAl(111), ITizAl(201) des pics de Bragg de TiAl et Ti3Al et de l'intensité du halo de la phase amorphe lam, les fractions volumiques de $\mathrm{TiAl} \mathrm{Ti}_{3} \mathrm{Al}$ et de la phase amorphe ont été estimées. Les proportions relatives de TiAl et $\mathrm{Ti}_{3} \mathrm{Al}$ ont été obtenues à partir de l'équation [12]:

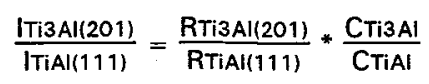

où ITizal(201) et ItiAl(111) sont les intensites des pics de Bragg (201) de TizAl et (111) de TiAl, CTi3Al et CTiAl sont les fractions volumiques de $\mathrm{Ti}_{3} \mathrm{Al}$ et TiAl. R est une fonction du volume de la maille élémentaire (v), du facteur de structure (F), du facteur de multiplicité (p), de l'angle de Bragg ( $\theta$ ), du facteur de température $\exp (-2 M)$ :

$$
R=\frac{1}{v^{2}}|F|^{2} p \frac{1+(\cos 2 \theta)^{2}}{\sin ^{2} \theta^{*} \cos \theta} \exp (-2 M)
$$

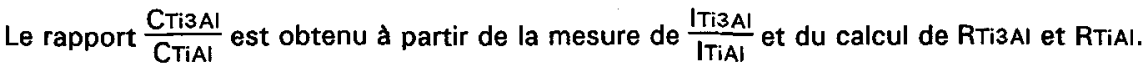

Les équations supplémentaires sont:

$C T i 3 A I+C T I A I=1$ quand il n'y a pas amorphisation

$\mathrm{CTT}_{\mathrm{i}} \mathrm{Al}+\mathrm{CTIAl}+\mathrm{C}_{\mathrm{am}}=1$ quand il y a amorphisation

Cam est obtenue par l'équation :

$$
\mathrm{Cam}=\frac{\operatorname{lam}}{\operatorname{lam}+\text { lorist }}
$$

Icrist est l'intensité des phases cristallines dans le domaine angulaire de la phase amorphe.

La figure 3 montre l'évolution des fractions volumiques des phases TiAl, TizAl et amorphe en fonction de la duree de broyage. La structure initiale est dominée par TizAl. Après 6 minutes de broyage, la fraction volumique de $\mathrm{Ti}_{3} \mathrm{Al}$ décroît au profit de la phase TiAl. La fraction de TiAl augmente après 15 minutes de broyage et reste à peu près constante jusqu'à 1 heure. Après 2 heures, la fraction volumique de TiAl décroît alors que celle de $\mathrm{Ti}_{3} \mathrm{Al}$ augmente. Une phase amorphe est détectée, la fraction volumique de cette phase est 0.29 et 0.66 respectivement après 2 heures et 4 heures de broyage.

La méthode de la largeur intégrale [13] a été appliquée pour déterminer la taille des cristallites lou domaines cohérents) et la déformation élastique interne qui contribuent à l'élargissement des pics (corrigé de l'élargissement de raie instrumental). Cette méthode consiste à tracer $\frac{\beta^{2}}{(\tan \theta)^{2}}$ en fonction de $\frac{\beta}{\sin \theta \tan \theta}, \beta$ est la largeur à mi-hauteur, $\theta$ est l'angle de Bragg. Cette méthode n'est plus valable pour des domaines cohérents de taille supérieure à $200 \mathrm{~nm}$. La phase TiAl présente peu de pics de Bragg aussi la méthode n'est plus valable pour des temps de broyage supérieurs a 15 minutes. Alors, la taille des cristallites est déterminée par la formule de Scherrer $t=\frac{\lambda}{\beta \cos \theta}\left(\lambda\right.$ est la longueur $d^{\prime}$ onde) appliquée au pic (111). Ainsi, il a été mis en évidence une diminution de la taille des cristallites de Tijal et TiAl jusqu'à $15-20 \mathrm{~nm}$ dès le début du broyage $\left(6\right.$ minutes) et une augmentation de la déformation élastique interne de la phase $\mathrm{Ti}_{3} \mathrm{Al}^{\mathrm{jusqu}} \mathrm{a}$ 
$6.3 \times 10^{-3}$ après 4 heures de broyage (annexe fig.5 et 6 ). II n'a pas été possible de mesurer correctement la déformation dans TiAl après 15 minutes de broyage.

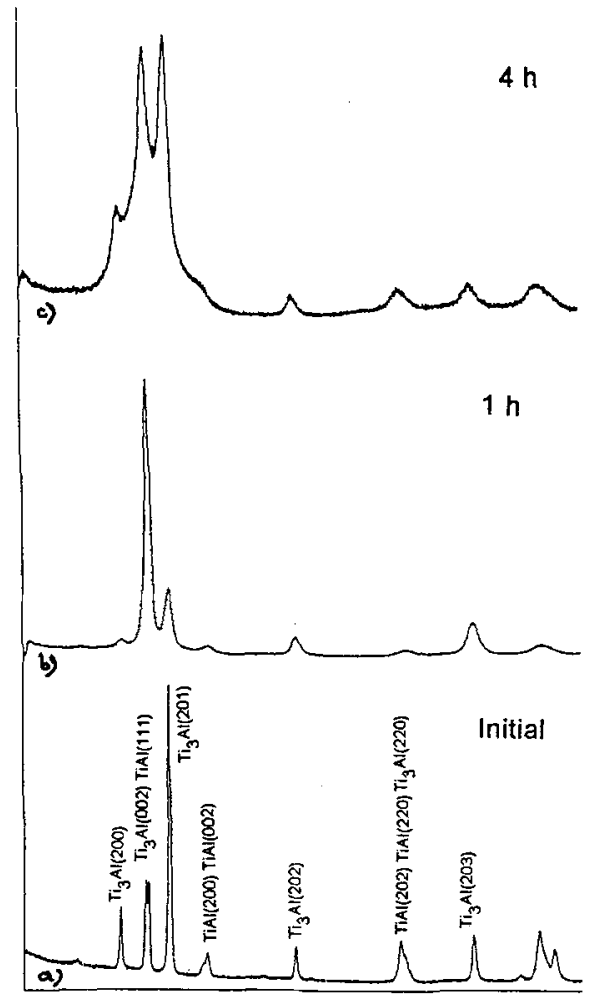

Fig. 2 - Spectres de diffraction $R X$ correspondant à a) initial, b) $1 \mathrm{~h}$, c) $4 \mathrm{~h}$ de broyage

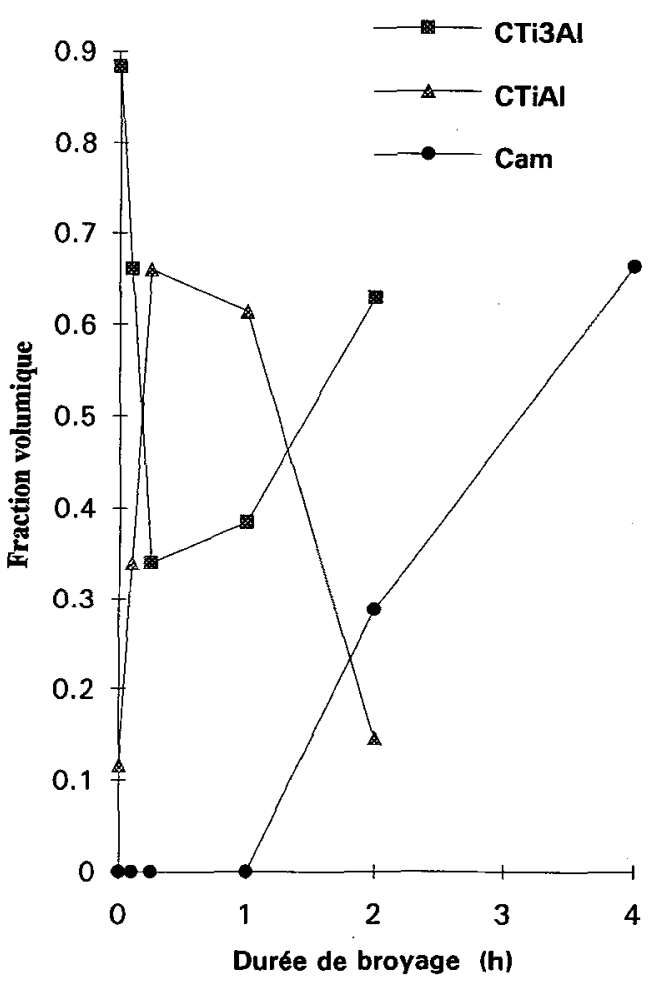

Fig. 3 - Fractions volumiques de phases $\mathrm{Ti}_{3} \mathrm{Al}$, $\mathrm{TiAl}$ et amorphes en fonction de la durée de broyage

\section{Discussion}

La poudre initiale Ti-47.5Al-3Cr (at.\%) présente une structure $\gamma+\alpha_{2}$ dominée par la phase TizAl. La composition de la phase $\mathrm{Ti}_{3} \mathrm{Al}$ est proche de la composition de la phase TiAl. Pour la poudre initiale, les paramètres de maille (exprimés en $\AA$ ) de la phase $\mathrm{Ti}_{3} \mathrm{Al}$ sont: $a=5.72$ and $c=4.59$. Le parametre a est supérieur à celui de $\mathrm{Ti}_{3} \mathrm{Al}$ équiatomique $(a=5.69, c=4.60)$ du fait d'un enrichissement en aluminium. La phase TiAl contient du chrome qui fait décroitre le paramètre de maille $c=4.03$ comparé à celui de TiAl équiatomique $(a=3.99, c=4.06)$.

La structure hors équilibre $\gamma+\alpha_{2}$, dominée par $\mathrm{Ti}_{3} \mathrm{Al}$, est due à la solidification rapide imposée par l'atomisation gazeuse. Graves et al. [14] ont montré les limites des cinétiques de la croissance de composés intermétalliques au cours de la solidification rapide de TIAI: la phase $\alpha$-Ti (hcpl métastable est formée plus rapidement que la phase TiAl. La mise en ordre de la phase $\alpha$-Ti en $\mathrm{Ti}_{3} \mathrm{Al}$ a lieu à l'état solide, le domaine de la phase $\mathrm{Ti}_{3} \mathrm{Al}$ étant étendu jusqu'à la composition équiatomique. Une structure biphasée $\gamma+\alpha_{2}$ est obtenue dans la poudre, les proportions relatives de chaque phase dépendant de la taille des particules.

De plus, la solidification initiale de la phase $\alpha$ devrait être prévue par le diagramme binaire d'équilibre de phases. La transformation a l'état solide attendue $\alpha \rightarrow \alpha_{2}+\gamma$ est facile à supprimer lors d'un procédé de solidification rapide, laissant ainsi ta phase $\alpha$ métastable et les ségrégations interdendritiques initiales. La 
mécanosynthèse conduit à une homogénéisation de la structure de solidification initiale contenant des ségrégations à la fois grâce à des cycles de soudage et fracture et à la réduction de la taille des particules.

Nous avons observé que la mécanosynthèse de l'alliage Ti-47.5Al-3Cr (at.\%) conduit à la transformation partielle de la phase $\mathrm{Ti}_{3} \mathrm{Al}$ métastable en la phase d'équilibre TiAl lors des premiers temps de broyage. La transformation de $\alpha_{2}$ en $\gamma$ au début du broyage est un exemple de formation d'une structure ordonnée $(\gamma)$ à partir d'une structure métastable $\left(\alpha_{2}\right)$ lors du broyage. Cette transformation est certainement favorisée par la mobilité atomique causée par l'accroissement de la déformation et de la température au cours du broyage. Pour des durées de broyage supérieurs a 1 heure, l'amorphisation partielle a été mise en évidence par diffraction RX. Les analyses par diffraction RX ont aussi montré un élargissement des pics expliqué par la diminution de la taille des cristallites et par les défauts et contraintes induits dans les matériaux lors du broyage. L'affinement des cristallites a été confirmé en champ sombre au MET. Les clichés de diffraction indiquent une structure microcristallisée dans les poudres broyées et l'existence d'une phase amorphe dans la poudre broyée 4 heures. La microstructure ultrafine est produite par la déformation plastique élevée lors du brovage.

Les petits précipités ( $20 \mathrm{~nm}$ ) observés au MET dans les poudres broyées sont certainement des carbures ou des oxydes formés lors de la mécanosynthèse du fait de l'addition d'acide stéarique. Cette hypothèse doit encore être confirmée. Ces précipités devraient permettre le blocage des joints de grain et éviter le grossissement de grain.

Plusieurs interprétations pourraient expliquer la formation d'une phase amorphe après 2 heures de broyage et l'état hors d'équilibre. Lors du broyage, la multiplication des joints de grain et l'augmentation des contraintes internes font croitre l'énergie des phases TiAl et Ti3 Al. Ces phases sont des composés intermétalliques qui peuvent emmagasiner de l'énergie sous forme de désordre atomique: les anti-sites et les parois d'antiphase sont générés lors du broyage et cette énergie est probablement supérieure à l'énergie des autres défauts. Si l'accroissement de l'énergie est suffisant, les phases amorphes et métastables peuvent se former $[15,16]$. Les différentes contributions énergétiques doivent être évaluées et ensuite analysées à l'aide du diagramme d'énergie libre. Une autre approche est celle de G. Martin et al. qui considerent la thermodynamique sous contrainte avec déplacements atomiques athermiques lors des chocs. Ils établissent ainsi des diagrammes de phase à l'équilibre dynamique. Ils ne parlent plus d'énergie libre car ce terme ne se justifie que pour des transitions de phases avec déplacements atomiques activés thermiquement [17]. Les résultats de notre étude doivent être complétés par des dosages d'oxygène et de fer pour s'assurer que ces éléments n'interfèrent pas dans le mécanisme d'amorphisation.

\section{Conclusion}

La mécanosynthèse de la poudre pré-alliée Ti-47.5Al-3Cr (at. \%) conduit à la transformation partielle de la phase métastable Ti3 $\mathrm{Al}$ en la phase d'équilibre TiAl. L'amorphisation partielle cornmence après 2 heures de broyage. Le broyage de la poudre pré-alliée $\mathrm{Ti}-47.5 \mathrm{Al}-3 \mathrm{Cr}$ solidifiée rapidement est un excellent exemple d'application de la mécanosynthèse commme moyen de modifier et de sélectionner une phase par un procédé de transformation à l'état solide, partant d'une structure de solidification métastable $\alpha_{2}+\gamma$ avec ségrégations pour aller vers une structure d'équilibre, la phase $\gamma$, puis retour vers la phase $\alpha_{2}$ homogène et métastable et/ou une phase amorphe.

Une microstructure ultrafine est obtenue avec des tailles de cristallites de 10 à $15 \mathrm{~nm}$.

Des particules nanométriques $(-20 \mathrm{~nm})$ sont observées dans les poudres broyées. Ces particules sont certainement formées par réaction de l'alliage avec l'adjuvant (acide stéarique).

\section{Remerciements}

Les auteurs remercient le Professeur P.Desré de I'Institut National Polytechnique de Grenoble, France, et le Docteur G.Martin, du CEREM Saclay, France, pour leur aide dans la compréhension des mécanismes intervenant lors de la mécanosynthèse de composé intermétalliques. Nous remercions aussi le Docteur. W.Wallace du National Research Council of Canada, Ottawa, Canada. 


\section{Références}

11/ S.C. Huang, E.L. Hall, Metallurgical Transactions A 22, 2619-2627 (1991)

12/ M. Kimura, K. Hashimoto, H. Morikawa, Mat. Sci. \&. Eng. A 152, 54-59 (1992)

13/ S. Naka, M. Thomas, T. Khan, Mat. Sci. \&.Tech. 8, 291-298 (1992)

14/ F.H. Froes, C. Suryanarayana, G.H. Chen, A. Frefer, G.R. Hyde, J.of Metals,.26-29 (1992)

/5/ J.S. Benjamin, Mat. Sci. For. vol. 88-90, 1-18 (1992)

16/ M.Bodart, D.Brenet, F.Moret, ASM Int. Conf. on Advanced Mat. and Processes, Paris, France, 11-13 September 1991

17/ A.Alamo, J.Decours, M.Pigoury, C.Foucher, Proc. Int. Conf. on Stuctural Applications of Mechanical Alloying, Myrtle Beach, Ed. F.H.Froes, J.J.DeBarbadillo (1990)

18/ G. Cocco, I. Soletta, L. Battezzati, M. Baricco, S. Enzo, Philosophical Magazine B, 61, N4, 473-486 (1990)

19/ C. Suryanarayana, R. Sundaresan, F.H. Froes, Mat. Sci. \& Eng. A 150, 117-121 (1992)

/10/G.H. Chen, C. Suryanarayana, F.H. Froes, Scr. Metal. \& Mater. 25, 2537-2540 (1991)

111/M.Bodart, F.Moret, R.Baccino, Powder Metallurgy World Congress, San Francisco, California, USA, 2126 June 1992

112/B.D.Cullity, "Elements of X-ray diffraction", Addison-Wesley, New York (1978)

/13/H.Klugg, L.Alexander, "X-ray diffraction procedures", Wiley (1974)

114/ J.A. Graves, J.H. Perepezko, C.H. Ward, F.H. Froes, Scr. Metallurgica 21, 567-572 (1987)

/15/C.C.Koch, "Mechanical Milling and Alloying" Mat. Sci. \&. Tech. A Comprehensive Treatment, vol.15 p 193, Ed. R.W.Cahn, P.Naasen, Weinheim, New York (1991)

/16/L.M.Di, "Mechanically induced atomic disorder and phase transformations", thèse, Amsterdam, The Netherlands (1992)

/17/P.Bellon, G. Martin, Proc. Ordering and Disordering in Alloys, Grenoble, France, 10-12 July 1991 


\section{Annexe}

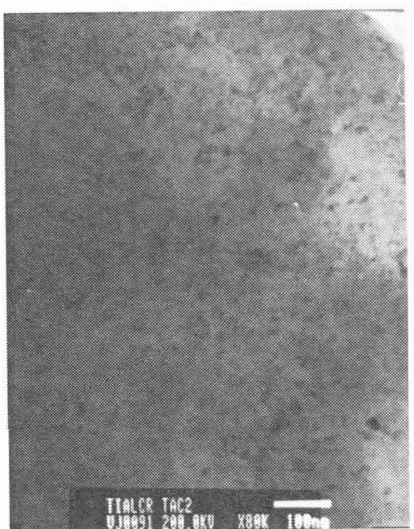

a) Champ clair

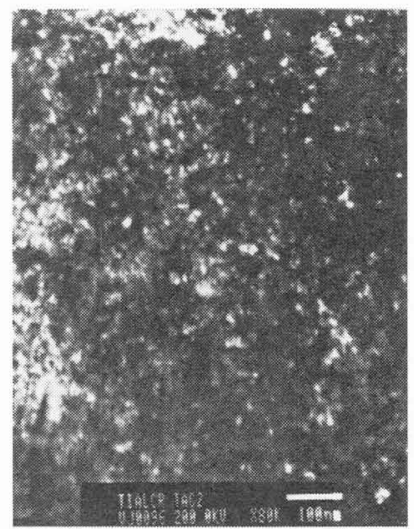

b) Champ sombre

Fig 1 - TEM. Poudre broyée 6 minutes

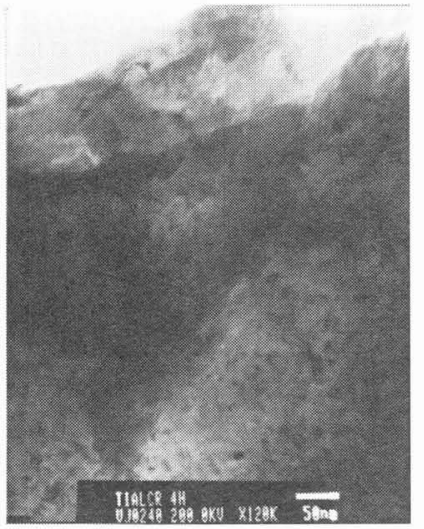

a) Champ clair

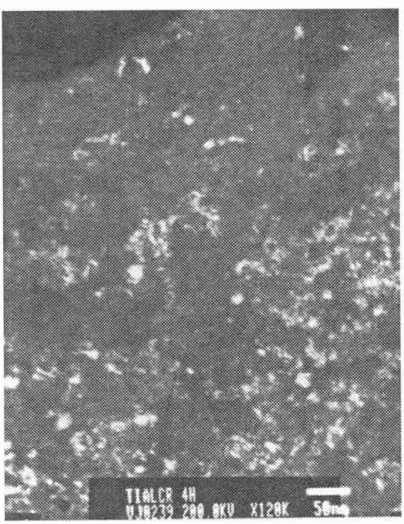

b) Champ sombre

Fig 2 - TEM. Poudre broyée 4 heures

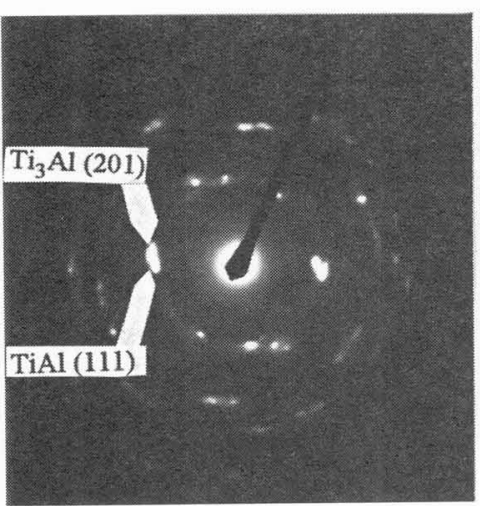

a) Poudre initiale

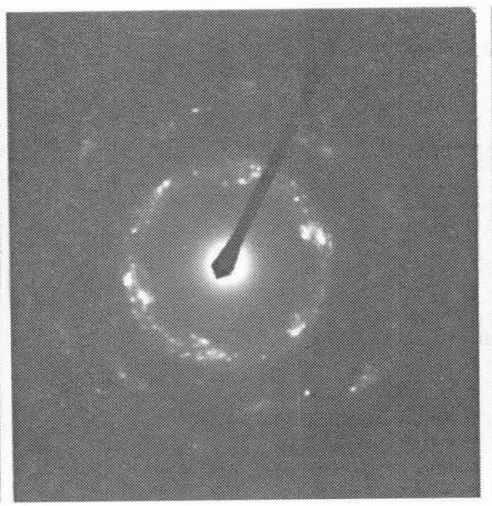

b) Poudre broyée $6 \mathrm{~min}$. Fig 3 - TEM. Clichés de diffraction

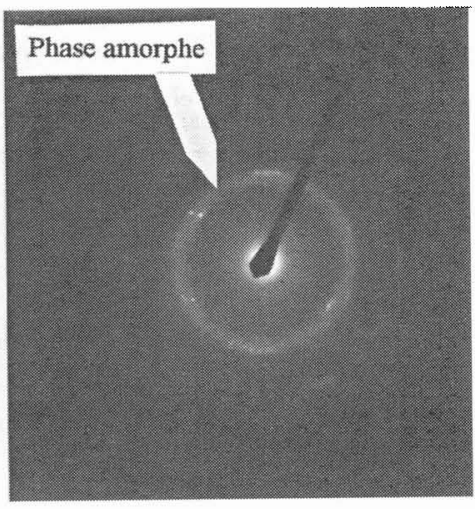

c) Poudre broyée 4h. 


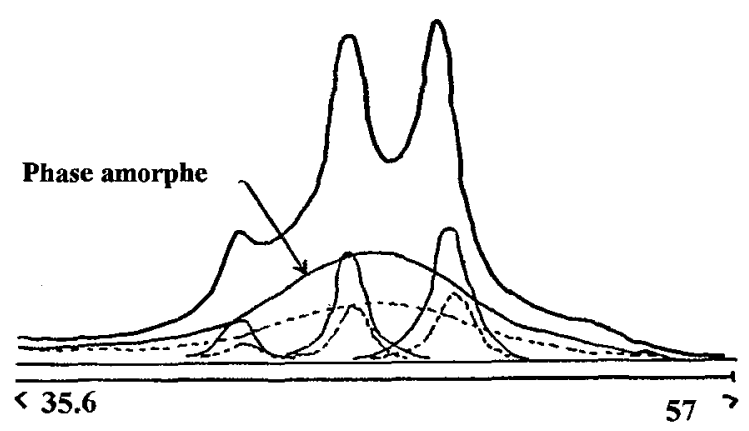

Fig 4 - Spectre de diffraction de RX de la poudre broyée 4 heures après déconvolution

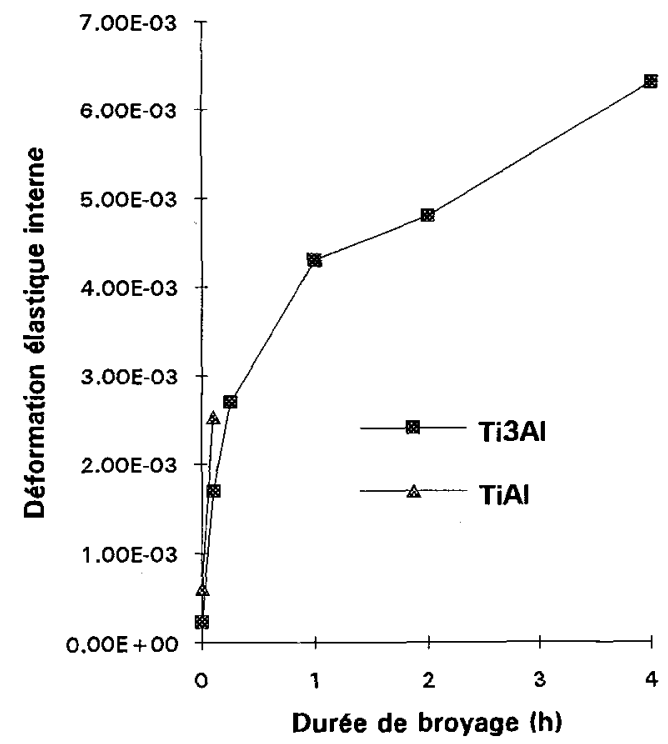

Fig 5 - Taille des cristallites en fonction de la durée de broyage

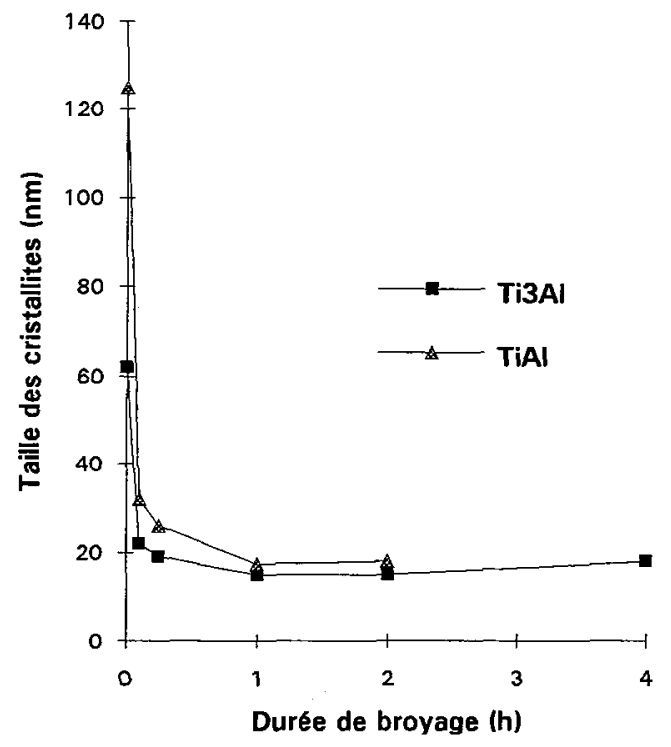

Fig 6 - Déformation élastique interne en fonction de la durée de broyage 